\title{
DECONFINEMENT AND VIRTUAL QUARK LOOPS
}

\author{
T. ÇELIK ${ }^{1}$, J. ENGELS and H. SATZ \\ Fakultät für Physik, Universität Bielefeld, Germany
}

Received 5 September 1983

Revised manuscript received 19 October 1983

\begin{abstract}
We calculate per Monte Carlo evaluation on an $8^{3} \times 3$ lattice the energy density $\epsilon_{\mathrm{G}}$ of the gluon sector of QCD, including virtual quark loops up to the fourth power in the hopping parameter expansion. For light quarks of one flavour, we observe at $T / \Lambda_{\mathrm{L}} \simeq 95 \pm 10$ a rapid variation of $\epsilon_{\mathrm{G}}$ in $T$, accompanied by strong fluctuations from iteration to iteration, as clear signal of the deconfinement transition.
\end{abstract}

The deconfinement transition, at which strongly interacting matter becomes a colour-conducting plasma, has so far been studied for pure Yang-Mills systems and for full QCD without virtual quark loops ("quenched approximation"). The concentration on Yang-Mills fields in the first studies of the phenomenon is physically meaningful: non-abelian gauge fields alone already exhibit confinement at low $[1,2]$ and deconfinement at high [3] temperatures, at least on the lattice. The statistical mechanics of $\mathrm{SU}(N)$ gauge fields ${ }^{\neq 1}$ has therefore contributed significantly to our understanding of colour force thermodynamics. On the other hand, the neglect of virtual quark loops in the extension of full QCD is due to technical difficulties rather than to physical reasoning: the evaluation of the $r \times r$ fermion matrix, where $r$ is some multiple of the number of lattice sites (typically 10000 or more), quickly led to the limits of computer possibilities both in speed and in memory space. Nevertheless, it is the virtual quark loops which provide the "breaking of the string" through meson formation, so their role in deconfinement is certainly crucial. The advent of array processors has now put the inclusion of fermions within reach, and the aim of this paper is to present first results on the statistical mechanics of

\footnotetext{
1 Alexander von Humboldt fellow, on leave from Hacettepe University, Ankara, Turkey.

$\neq 1$ For a survey see ref. [4].
}

QCD with virtual quark loops. More precisely, we shall show how the energy density $\epsilon_{\mathrm{G}}$ of the gluon sector of QCD is modified when virtual quarks are included. Pure SU(3) gauge theory, we recall, led to a first order phase transition at the deconfinement point [5], and we want to see what happens to this when light virtual quarks of one flavour are brought in.

A phase transition can be studied in two ways. We may consider some thermodynamic quantity - energy density, specific heat - and look for a discontinuity or singularity; or we may construct a specific order parameter to distinguish the two phases. Thus, in the Ising model, the Curie point can be characterized either by the singularity of the specific heat or through the vanishing of the spontaneous magnetization.

In SU( $N)$ Yang-Mills theory, deconfinement is related to the breaking of a global $Z_{N}$ symmetry [6,7]. The euclidean formulation of the partition function [8] requires the gauge fields to be periodic at the boundaries of the imaginary time integration range: $A(x, 0)=A(x, \beta)$, where $\beta=T^{-1}$ is the inverse physical temperature. On a lattice with spacing $a$, this implies that gauge transformations $V_{\boldsymbol{x}, \tau}$ must satisfy

$V_{\boldsymbol{x}, \tau=0}=V_{\boldsymbol{x}, \tau=N_{\beta}} \quad \forall \boldsymbol{x}$,

with $\beta=N_{\beta} a$ for the temporal lattice axis. The Yang-Mills action, because it consists of closed loops, is invariant under the larger class of transformations 
$V_{x, \tau=0}=c_{N} V_{x, \tau=N_{\beta}} \quad \forall \boldsymbol{x}$,

where $c_{N} 1 \in \mathrm{Z}_{N}$ is an element of the center $\mathrm{Z}_{N}$ of the $\mathrm{SU}(N)$ gauge group. On the other hand, the thermal Wilson loop

$L(\boldsymbol{x}) \equiv \operatorname{tr} \prod_{\tau=1}^{N_{\beta}} U_{\boldsymbol{x} ; \tau, \tau+1}$,

where $U_{x ; \tau, \tau+1}$ is the gauge group element on the link connecting $\tau$ and $\tau+1$ at $x$, is not invariant under the transformation (2): it becomes

$L^{\prime}(x)=c_{N} L(x)$.

The expectation value $\langle L\rangle$, averaged over $\boldsymbol{x}$, thus constitutes an order parameter of the state. Since $\langle L\rangle$ is connected to the free energy $F_{\mathrm{Q}}$ of an isolated colour source [6],

$\langle L\rangle \sim \exp \left(-\beta F_{\mathrm{Q}}\right)$,

it vanishes in the confined state and becomes non-zero after deconfinement.

The introduction of fermions into the action breaks the global invariance just discussed. In the euclidean form, fermion fields must be antiperiodic at the $\tau$ boundary, and hence the action contains terms of the type

$$
\begin{gathered}
\bar{\psi}_{\boldsymbol{x}, N_{\beta}-1} U_{\boldsymbol{x} ; N_{\beta}-1, N_{\beta}} \psi_{\boldsymbol{x}, N_{\beta}}=-\bar{\psi}_{\boldsymbol{x}, N_{\beta}-1} U_{\boldsymbol{x} ; N_{\beta}-1, N_{\beta}} \psi_{x, 0} \\
\rightarrow-c_{N}^{*} \bar{\psi}_{\boldsymbol{x}, N_{\beta}-1} U_{\boldsymbol{x} ; N_{\beta}-1, N_{\beta}} \psi_{\boldsymbol{x} ; 0} .
\end{gathered}
$$

It is thus evident that deconfinement in full QCD cannot be characterized in terms of a global invariance under the center of the gauge group, and in particular, that $\langle L\rangle$ no longer constitutes an order parameter distinguishing confined and deconfined states. We shall therefore follow the alternative approach to transition phenomena and look for singular behaviour of thermodynamic quantities.

Our starting point is the effective boson form of the euclidean partition function of QCD [9],

$Z_{\mathrm{E}}(\beta)=\int \prod_{\text {links }} \mathrm{d} U \operatorname{det}(1-\kappa M) \exp \left[-S_{\mathrm{G}}(U)\right]$,

where $S_{\mathrm{G}}(U)$ is the usual Wilson action for SU(3) gauge fields at finite temperature,

$$
\begin{aligned}
& S_{\mathrm{G}}(U)=6\left(g_{\beta}^{-2}\left(a_{\sigma} / a_{\beta}\right) \sum_{\mathrm{P}_{\beta}}\left(1-\frac{1}{3} \operatorname{Re} \operatorname{tr} U U U U\right)\right. \\
& \left.+g_{\sigma}^{-2}\left(a_{\beta} / a_{\sigma}\right) \sum_{\mathbf{P}_{\sigma}}\left(1-\frac{1}{3} \operatorname{Re} \operatorname{tr} U U U U\right)\right),
\end{aligned}
$$

obtained by summing over space-like $\left(\mathrm{P}_{\sigma}\right)$ and spacetime $\left(\mathrm{P}_{\beta}\right)$ plaquettes; $\mathrm{a}_{\sigma}$ and $\mathrm{a}_{\beta}$ are the spatial and temporal lattice spacings, $g_{\sigma}$ and $g_{\beta}$ the corresponding couplings. The matrix $M$,

$M_{\mu}(U)=\left(1-\gamma_{\mu}\right) U_{n m} \delta_{n, m-\hat{\mu}}+\left(1+\gamma_{\mu}\right) U_{m n}^{+} \delta_{n, m+\hat{\mu}}$

describes the interaction between fermions of one flavour, corresponding to the Wilson form

$S_{\mathrm{F}}(U)=\bar{\psi}(1-\kappa M) \psi$.

In the finite temperature case, the hopping parameter $\kappa\left(g^{2}\right)$ is "link-direction" dependent:

$\kappa M=\kappa_{\beta} M_{0}+\kappa_{\sigma} \sum_{\mu=1}^{3} M_{\mu}$.

The resulting euclidean energy density of the gluon sector, obtained from

$$
\begin{aligned}
\epsilon_{\mathrm{G}}^{\mathrm{E}} & =\frac{1}{Z_{\mathrm{E}} N_{\sigma}^{3} N_{\beta} a_{\sigma}^{3} a_{\beta}} \int \prod_{\text {links }} \mathrm{d} U \operatorname{det}(1-\kappa M) \\
& \times \exp \left[-S_{\mathrm{G}}(U)\right]\left(\partial S_{\mathrm{G}}(U) / \partial a_{\beta}\right)_{a_{\sigma}},
\end{aligned}
$$

with $N_{\sigma}\left(N_{\beta}\right)$ denoting the number of lattice sites per spatial (temporal) axis, leads for $a_{\sigma}=a_{\beta}=a$ (where $g_{\sigma}$ $\left.=g_{\beta}=g, \kappa_{\sigma}=\kappa_{\beta}=\kappa\right)$ to the physical energy density

$$
\begin{aligned}
& \epsilon_{\mathrm{G}} / T^{4}=18 N_{\beta}^{4}\left[\left(g^{-2}\right)\left(\bar{P}_{\sigma}-\bar{P}_{\beta}\right)+c_{\beta}^{\prime}\left(\bar{P}-\bar{P}_{\sigma}\right)\right. \\
& \left.\quad+c_{\beta}^{\prime}\left(\bar{P}-\bar{P}_{\beta}\right)\right],
\end{aligned}
$$

where $\bar{P}_{\sigma}, \bar{P}_{\beta}$ and $\bar{P}$ are the lattice expectation values for space-like, space-time and symmetric lattice plaquettes, respectively. The averaging here is carried out with both the gluon Boltzmann factor and the fermion determinant as weights,

$\widetilde{P}_{\sigma}=\frac{1}{Z_{\mathrm{E}}} \int \prod_{\text {links }} \mathrm{d} U \operatorname{det}(1-\kappa M) \exp \left[-S_{\mathrm{G}}(U)\right]$

$\sum_{\mathrm{P}_{\sigma}}\left(1-\frac{1}{3} \operatorname{Re} \operatorname{tr} U U U U\right) / \sum_{\mathrm{P}_{\sigma}}$

and similarly for $\bar{P}_{\beta}$ and $\bar{P}$; the factor $\operatorname{det}(1-\kappa M)$ 
provides the effect of virtual quark loops on the gluon system. The constants $c_{\sigma}^{\prime}$ and $c_{\beta}^{\prime}$ result from the differentiation of the couplings $g_{\sigma}$ and $g_{\beta}$ with respect to $a_{\beta}$; they are known, and for $a_{\sigma}=a_{\beta}$ and colour SU(3) one has [10] $c_{\sigma}^{\prime}=0.197633, c_{\beta}^{\prime}=-0.132203$. The problem is thus one of evaluating plaquette averages in the presence of $\operatorname{det}(1-\kappa M)$; in the quenched approximation, this determinant is set equal to unity and hence the effect of virtual quark loops on the gluon system is neglected.

We now expand the logarithm of $\operatorname{det}(1-\kappa M)$ in powers of the hopping parameter $\kappa\left(g^{2}\right)$

$\ln \operatorname{det}(1-\kappa M)=-\operatorname{tr} \sum_{l=1}^{\infty} \frac{\kappa^{l}}{l} M^{l}$.

Only closed loops contribute to the right-hand side of eq. (15). Taking into account the determinant in the Monte Carlo update of the links corresponds now to replacing $S_{\mathrm{G}}(U)$ by a new effective action $S_{\text {eff }}(U)$. On an $N_{\sigma}^{3} \times N_{\beta}$ lattice with $N_{\beta}=3$,

$$
\begin{aligned}
S_{\text {eff }} & =S_{\mathrm{G}}(U)-32 \kappa^{3} \sum_{\text {sites }} \operatorname{Re} L \\
& -16 \kappa^{4} \sum_{P_{\sigma}, P_{\beta}} \operatorname{Re} \operatorname{tr} U U U U+O\left(\kappa^{5}\right),
\end{aligned}
$$

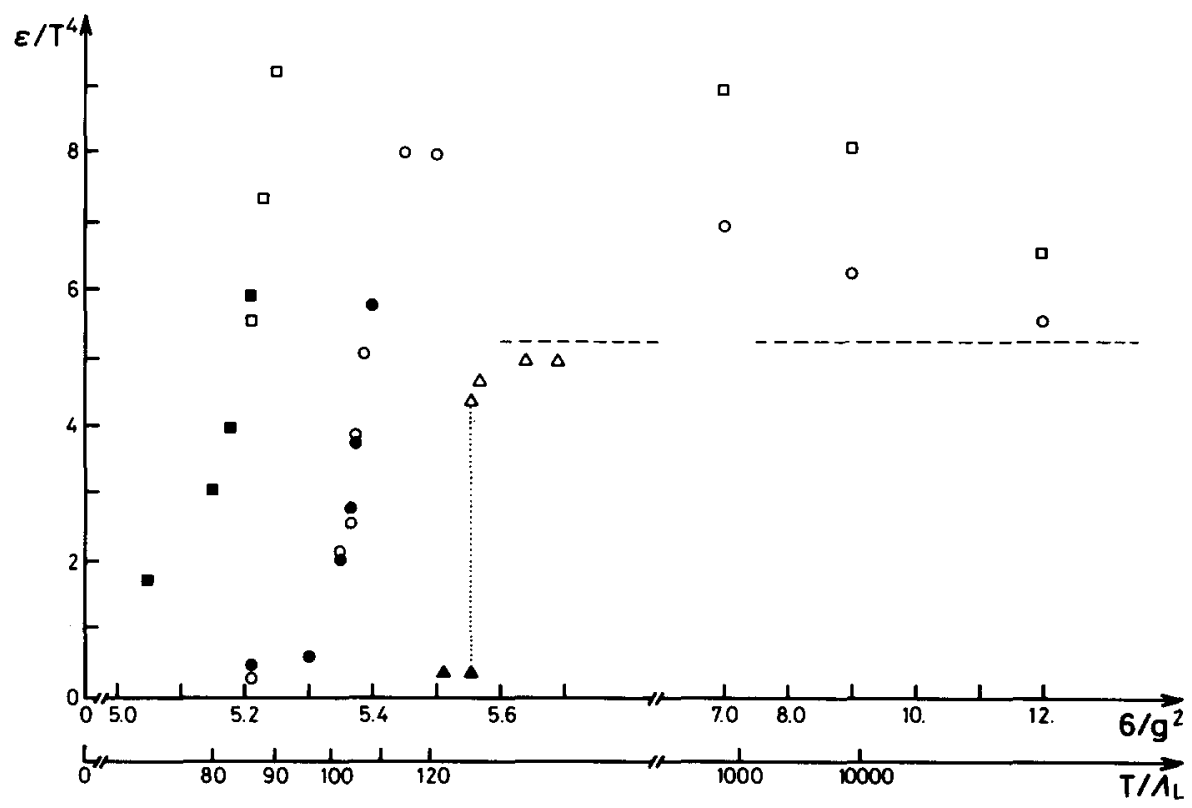

Fig. 1. Gluon energy density $\epsilon_{\mathrm{G}} / T^{4}$ in fourth order hopping parameter expansion as function of $6 / g^{2}$ and $T / \Lambda_{\mathrm{L}}$, for $\kappa=0.20$ ( $\square$ ordered, - disordered start), $\kappa=0.15$ (o ordered, $\bullet$ disordered start) and $\kappa=0\left(\triangle\right.$ ordered, $\wedge$ disordered start; here the $T / \Lambda_{\mathrm{L}}$ scale does not apply). The dashed line is the ideal gas limit.

where, because of periodicity in the temporal direction, the lowest order contribution in $k$ is given by the thermal Wilson loop. For the present, we include only terms up to fourth order in $\kappa$.

To calculate $\epsilon_{\mathrm{G}}(\beta)$, we still need to know the value of the hopping parameter $\kappa\left(g^{2}\right)$ at given $g^{2}$, for massless quarks. At small $g^{2}, k$ is approximated by [11]

$\kappa\left(g^{2}\right) \simeq \frac{1}{8}\left(1+0.099 g^{2}\right)$.

At larger $g^{2}$, there are Monte Carlo results [12] for the region between the strong coupling value $k=0.25$ and the weak limit $\kappa=1 / 8$. For the $g^{2}$ range we are interested in, we take $\kappa=0.15$ and $\kappa=0.20$ as indicative values.

As we shall here calculate only $\bar{P}_{\sigma}$ and $\bar{P}_{\beta}$, a comment is needed on the $\bar{P}$ values used. The inclusion of the fermion determinant up to fourth order in the hopping parameter expansion simply means shifting

$\left(6 / g^{2}\right) \rightarrow\left(6 / g^{2}\right)+48 \kappa^{4}$

in the evaluation of $\bar{P}$, where no $n=3$ loop exists. With this shift, we use the values of $\bar{P}$ obtained in ref. [13].

In fig. 1, we now show the energy density (13), evaluated on an $8^{3} \times 3$ lattice, as function of $6 / g^{2}$. Our results are normalized to the Stefan-Boltzmann

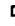


form $\epsilon_{\mathrm{G}}^{\mathrm{SB}}$ for a non-interacting gluon system, evaluated also on an $8^{3} \times 3$ lattice [14]. To obtain $\epsilon_{\mathrm{G}}$ as function of $T=\left(N_{\beta} a\right)^{-1}$, we use the normalization group relation

$$
\begin{aligned}
& a \Lambda_{\mathrm{L}}=\exp \left\{-\frac{4 \pi^{2}}{33-2 N_{\mathrm{f}}}\left(\frac{6}{g^{2}}\right)\right. \\
& \left.+\frac{459-57 N_{\mathrm{f}}}{\left(33-2 N_{\mathrm{f}}\right)^{2}} \ln \left[\frac{8 \pi^{2}}{33-2 N_{\mathrm{f}}}\left(\frac{6}{g^{2}}\right)\right]\right\},
\end{aligned}
$$

with $N_{\mathrm{f}}=1$. We note that $\epsilon_{\mathrm{G}}$ shows an rapid rise at $6 / g^{2} \simeq 5.38$ for $\kappa=0.15$ and at $6 / g^{2} \simeq 5.24$ for $\kappa$ $=0.20$; hence the specific heat $\mathrm{c}_{\mathrm{V}}=(\partial \epsilon / \partial T) \sim(\partial \epsilon /$ $\partial g^{-2}$ ), as shown in fig. 2 , has a singularity-like peak there, as a clear signal of deconfinement. Using eq. (19), the corresponding critical temperature $T_{\mathrm{c}} / \Lambda_{\mathrm{L}}$ becomes 89 for $\kappa=0.15$ and 105 for $\kappa=0.20$; this suggests a value of $95 \pm 10$, to be compared to 86 for the Yang-Mills system [5].

These results are obtained in fourth order of the $\kappa$-expansion. The effect of higher order terms is under investigation, but we note here already that the fourth order term only provides, by eq. (18), for fig. 1 an overall shift in $6 / g^{2}$ by 0.024 for $k=0.15$ and by 0.072 for $\kappa=0.2$.

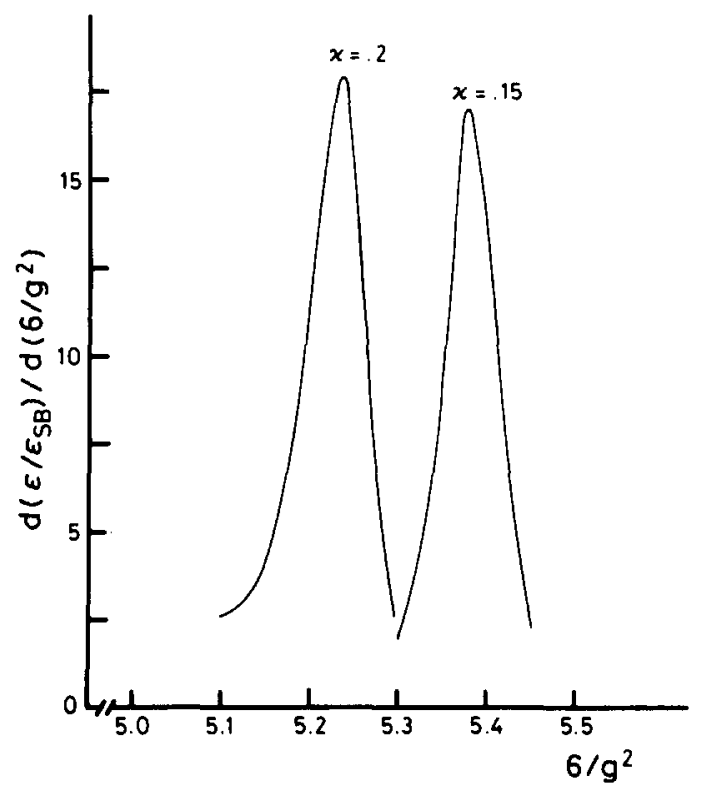

Fig. 2. The derivative $d\left(\epsilon_{\mathrm{G}} / \epsilon_{\mathrm{G}} \mathrm{SB}\right) / \mathrm{d}\left(6 / g^{2}\right) \sim c \mathrm{~V}$, as function of $6 / g^{2}$

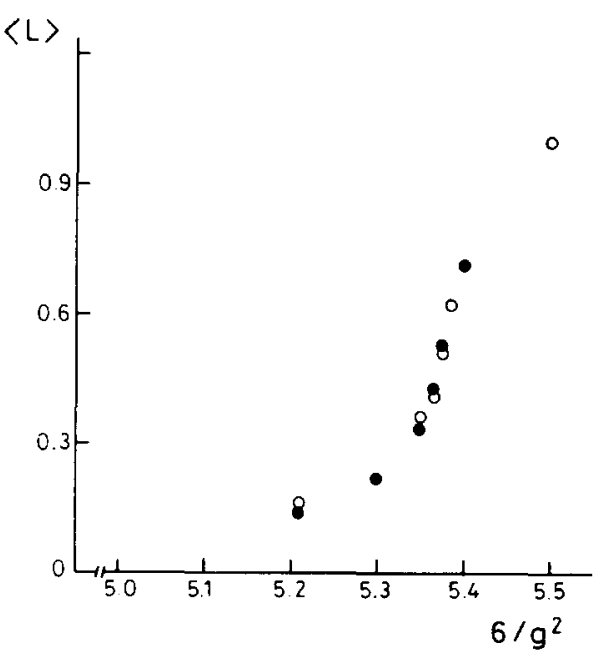

Fig. 3. Average thermal Wilson loop $\langle L\rangle$ for $\kappa=0.15$ as function of $6 / g^{2}$ ( $\circ$ ordered, $\bullet$ disordered start).

In contrast to the abrupt change of $\epsilon_{\mathrm{G}}$ in the transition region, the thermal Wilson loop $\langle L\rangle$ does not vary greatly there, as seen in fig. 3 .

The behaviour of $\epsilon_{\mathrm{G}}$ found here is to be compared with the behaviour of the pure Yang-Mills systems for colour SU(3) and SU(2). We note that the first order transition observed for the SU(3) Yang-Mills system [5] appears to be transformed into a second order transition by the inclusion of virtual quark loops. On the other hand, the variation of $\epsilon_{\mathrm{G}}$ in fig. 1 appears still more rapid than the second order transition of the SU(2) Yang-Mills system [15]: in both cases, $\epsilon$ / $T^{4}$ requires about $20 \Lambda_{\mathrm{L}}$ to rise from "bottom to top"; but $\Lambda_{\mathrm{L}}^{\mathrm{SU}(2)} / \Lambda_{\mathrm{L}}^{\mathrm{SU}(3)} \simeq 2$. We note further that at high $T$ our $\epsilon_{\mathrm{G}}(T)$ approaches the Stefan-Boltzmann limit; but it does so much slower than for the pure YangMills systems, and from above.

To provide further evidence for the second order nature of the transition for the gluon system with quark loops, we show in fig. 4a the behaviour of $\epsilon_{\mathrm{G}}$ as function of the number of Monte Carlo iterations, starting ence from a completely ordered and once from a random initial configuration, in the vicinity of the deconfinement point. In fig. $4 \mathrm{~b}$, the corresponding behaviour is shown for the pure SU(3) Yang-Mills case While the latter shows a clear two-state signal, we find in fig. $4 \mathrm{a}$ the strong random fluctuations expected of a second order transition.

We are interested here in QCD with light quarks. To 


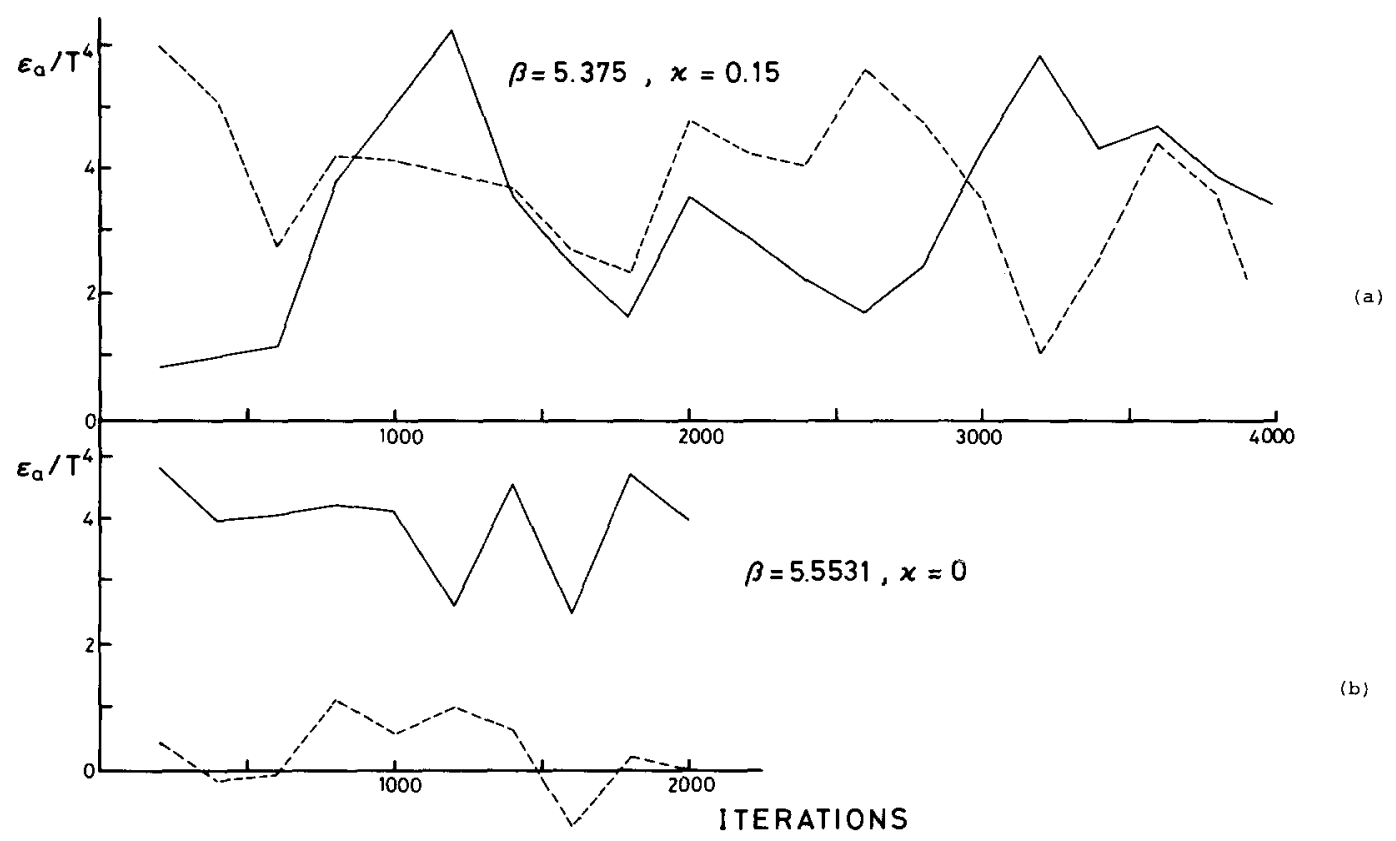

Fig. 4. (a) Gluon energy density $\epsilon_{\mathrm{G}} / T^{4}$ with quark loops, as function of the number of lattice iterations, in bins of 200 , for $\kappa$ $=0.15$, at the deconfinement point. (b) The corresponding gluon energy density $\epsilon_{\mathrm{G}} / T^{4}$ for the pure Yang-Mills system.

ensure a small quark mass, we must know rather precisely $\kappa_{c}$, the value of the hopping parameter at the pion pole, as function of $g^{2}$. Let us see what the uncertainties in $\kappa$ imply here. For the strong coupling value $\kappa_{\mathrm{c}}=0.25$, the proposed relation [12]

$\frac{1}{2}\left(1 / \kappa-1 / \kappa_{\mathrm{c}}\right)=\exp \left(m_{\mathrm{q}} a\right)-1$,

gives us as estimates $m_{\mathrm{q}}=2.5 T_{\mathrm{c}}$ for $\kappa=0.15$ and $m_{\mathrm{q}}$ $=1.2 T_{\mathrm{c}}$ for $\kappa=0.20$, in the case of one flavour. If $\kappa_{\mathrm{c}} \simeq 0.20$ [12], then $\kappa=0.15$ gives $m_{\mathrm{q}}=1.8 T_{\mathrm{c}}$, while the $\kappa=0.20$ curve corresponds to massless quarks.

Finally, we want to comment on recent conjectures that in the presence of virtual quark loops deconfinement no longer exists as a formal phase transition [16-18]. As noted above, fermions break the global symmetry of the action under the center of the gauge group; hence $\langle L\rangle$ can no longer be used to define confinement and deconfinement phases. Unless we find a new order parameter, we can thus only look if some thermodynamic function shows discontinuous or singular behaviour as signal of a phase transition.

Such a discontinuity can occur as a "continuation" of the Yang-Mills transition, if the latter is of first order [SU(3) gauge fields in three space dimensions].
Then only for sufficiently strong fermion coupling will the loops smooth out completely the discontinuity.

In the case of a second order transition in the Yang-Mills system [e.g. SU(2) gauge fields], any singular behaviour would have to be related to a new order parameter or symmetry.

In either case, the physical basis for deconfinement, the Debye screening of colour forces, appears to be unchanged. Hence we expect as before to find an abrupt change of behaviour at some $T_{\mathrm{c}}$, and this is borne out by our results.

In ref. [18], it is found for the SU(3) system that the discontinuities in $\langle L\rangle$ and $\epsilon_{\mathrm{G}}$ at $\kappa=0$ persist for a range of $\kappa$ values $\left(\kappa \lesssim 0.08\right.$ on an $8^{3} \times 2$ lattice for one flavour) and then disappear. It is now argued that this situation corresponds to a line of first order transitions, terminating in a second order point; it is not shown, however, that for $\kappa>0.08$ there are no second order transitions. On an $8^{3} \times 3$ lattice with one flavour, the end of the first order region would be expected to lie at $\kappa=0.15$, where we do in fact find second order behaviour $^{\ddagger 2}$. It persists, however, also for some range

$\neq 2$ We thank P. Hasenfratz for pointing out this agreement between the results of ref. [18] and ours. 
of $\kappa$ values beyond this point (see fig. 2), so that we find no evidence for any disappearance of the deconfinement transition.

Since the extension to $N_{\mathrm{f}}>1$ is accompanied by a decrease of $k$ at fixed $6 / g^{2}$, we do not expect these conclusions to be much changed for $N_{\mathrm{f}}=3$.

A further important issue is of course the validity of a truncated hopping parameter expansion for $\kappa$ values up to 0.20 . Preliminary results obtained by including the $\kappa^{5}$ contribution do not, however, lead to any striking changes.

With our present approximations, this appears to leave us with three possible scenarios:

(1) Deconfinement in the presence of light quarks is a second order phase transition and lies at the end of the line of first order transitions arising as $k$ is increased. We still find, with relation (20), an appearant quark mass of about $1-2 T_{\mathrm{c}}$, but this may well decrease for more accurate $\kappa_{c}$, on a larger lattice, in higher order of $\kappa$; moreover, eq. (20) may have to be modified to account for interactions.

(2) Deconfinement is generally a second order transition, but for sufficiently heavy quarks (i.e., sufficiently small $k$ ) it is triggered to occur prematurely as a first order transition.

(3) There is no more singular thermodynamic behaviour for $\kappa$ greater than the value $\bar{\kappa}$ associated with the endpoint, and the case of light quarks corresponds to $\kappa$-values larger than $\bar{\kappa}$. In this situation, deconfinement ceases to be a phase transition.

There are rather clear tests to distinguish between these alternatives. To establish the occurrence of a second order transition, we have to show that the peak in $c_{\mathrm{V}}$ increases with increasing lattice size. If there is a second order transition for one value $\kappa$ only, decreasing $k$ should lead to a first order transition, increasing $\kappa$ to non-singular behaviour. With present precision, we do not see any difference between $k$ $=0.15$ and $\kappa=0.20$. In addition, it should of course be checked that one is in the scaling region of $g^{2}$.

In summary: we conclude that deconfinement as a rapid change in physical regimes persists for SU(3) gauge fields at finite temperature, when virtual quark loops are included. The transition coupling $6 / g_{\mathrm{c}}^{2}$ on a $8^{3} \times 3$ lattice is shifted to lower values than found for the corresponding Yang-Mills system, so that the deconfinement temperature does not seem to be much modified. For the nature of deconfinement, our results support a second order phase transition.

It is a pleasure to thank R. Gavai, P. Hasenfratz and F. Karsch for useful discussions; we are grateful to P. Hasenfratz, F. Karsch and I. Stamatescu for providing us with a preliminary version of their results. Our calculations were carried out on the Cyber 205 of the Bochum University Computer Center, whose help is greatly appreciated.

\section{References}

[1] K. Wilson, Phys. Rev. D10 (1974) 2445.

[2] E.T. Tomboulis, Phys. Rev. Lett. 50 (1983) 885.

[3] C. Borgs and E. Seiler, Nucl. Phys. B215 [FS 7] (1983) 125.

[4] H. Satz, Phys. Rep. 88 (1982) 349.

[5] T. Celik, J. Engels and H. Satz, Phys. Lett. 125B (1983) 411 ;

J. Kogut, et al., Phys. Rev. Lett. 50 (1983) 393.

[6] L. McLerran and B. Svetitsky, Phys. Rev. D24 (1981) 450.

[7] B. Svetitsky and L.G. Yaffe, Nucl. Phys. B210 [FS 6] (1982) 423.

[8] C. Bernard, Phys. Rev. D9 (1974) 3312.

[9] J. Engels, F. Karsch and H. Satz, Phys. Lett. 113B (1982) 398.

[10] R.C. Trinchero, Nucl. Phys. B227 (1983) 61.

[11] N. Kawamoto, Nucl. Phys, B190 [FS 3] (1981) 617.

[12] A. Hasenfratz, P. Hasenfratz, Z. Kunszt and C.B. Lang, Phys. Lett. 110B (1982) 289;

P. Hasenfratz and I. Mont vay, Meson spectrum in quenched QCD on a $16^{4}$ lattice, DESY-preprint 83-072 (1983), and further references given there.

[13] M. Creutz and K. Moriarty, Phys. Rev. D26 (1981) 2166; K. Moriarty, private communication.

[14] J. Engels, F. Karsch and H. Satz, Nucl. Phys. B205 [FS 5] (1982) 239.

[15] J. Engels, F. Karsch, I, Montvay and H. Satz, Nucl. Phys. B205 [FS 5] (1982) 545.

[16] T. Banks and A. Ukawa, Nucl. Phys. B225 [FS 9] (1983) 145.

[17] T.A. De Grand and C.E. De Tar, Phase structure of QCD at high temperature with massive quarks and finite quark density, Utah-Colorado preprint UU HEP 83/8-COLO HEP 66 (1983).

[18] P. Hasenfratz, F. Karsch and I.O. Stamatescu, Phys. Lett. 133 (1983) 221. 